\title{
Comparative Study of DSR, OLSR and ZRP in MANET under Varying Pause Time and Packet Transmission Rate
}

\author{
Rajeev Paulus \\ Assistant. Prof. ECED. Teaching Assistant,ECED \\ SSET,SHIATS,Allahabad, SSET,SHIATS,Allahabad
}

\author{
Manish Kr.Tiwari \\ M.Tech student \\ SSET,SHIATS,Allahabad, SSET,SHIATS,Allahabad
}

\begin{abstract}
MANET is an infrastructure less network which consists of mobile nodes without central administration. It's routing algorithm should not only be capable of finding the shortest path between the source and destination, but it should also be adaptive, in terms of the changing state of the nodes, the changing load conditions of the network and also the changing state of the environment. In this paper a performance comparison of three popular mobile ad-hoc network routing protocols i.e. Dynamic Source Routing (DSR), Optimization Link State Routing (OLSR) and Zone Routing Protocol (ZRP) is presented with variable pause time and packet transmission rate. A network simulator QualNet 6.1 from scalable networks is used to evaluate the performance of these protocols. The performance analysis is based on different network metrics such as Average End to End delay (s), Average Jitter(s), Throughput and Packet delivery ratio.
\end{abstract}

\section{Keywords}

MANET, DSR, OLSR, ZRP, QualNet 6.1.

\section{INTRODUCTION}

Over recent times in most of the electronic gadgets like laptops, tablets, and mobile phones, there is often a requirement to set up a network to enable communication among some of these devices. For movable devices, a wireless network is often very suitable [1].Wireless networks are classified infrastructure, or adhoc infrastructure. Mobile Ad-hoc Network (MANET) is a temporary network that is designed for communication among mobile nodes, without any need for fixed or prespecified infrastructure. Each node here itself works like a router[1]. Application areas of MANET are very large viz. military operations, disaster managements, rescue operations, meetings and conferences, educational purposes and many more. Since, in MANET, the network topology changes frequently, due to the mobility of nodes. Therefore, routing becomes a challenging issue [3].In MANETs there are many routing protocol such as: AODV $[4,5]$, DYMO [6], DSR [7, 2, 15], OLSR [8, 12], ZRP [9, 11, 13], FSR
[10] etc. Among all AODV, DSR, DYMO and ZRP are well known popular routing protocols and have been standardized by the IETF MANET working group. The three most popular reactive routing protocols for MANETs namely Ad-Hoc On-demand Distance

Vector (AODV), Dynamic Source Routing (DSR) and Dynamic MANET On-demand (DYMO), find route only when node has data to send. It avoids the need of frequent link and route updates therefore substantially reduces energy consumption when the traffic load is light or the network mobility is high. All of the above discussed protocols are operating only in Network layer. This paper evaluates the performance comparison of DSR, OLSR and ZRP protocols under different network conditions. The rest of the paper is organized as follows: Section-2 Related works; Section-3 Overview of Routing Protocols; Section-4 Simulation Setup, Section-5 Results and Discussion and performance comparison graphs. Finally, Conclusion is presented in Section-6.

\section{RELATED WORK}

V. Sahu, et.al [1], analyzed the performance variation of AODV by changing some parassmeters such as nodedensity, pause time, and rate of transmission of packets. Effect of two pathloss models such as free space and two rays are used in a well known network simulator Qualnet 5.2. They analyzed various performance parameters such as throughput, number of bytes received, average end-toend delay, throughput and PDR as an important parameter to judge the performance of a network. Higher throughput and PDR meant better performance. However, jitter and end-to-end delay was low. They observed that the overall performance for AODV degraded as node density was increased.

S. R. Raju, et.al [10] proposed an algorithm to provide improved quality of service via hybrid routing protocol ZRP. They used QualNet version 4.5 and evaluated the performance in ZRP,AODV,DSR to compare QoS parameters viz., throughput, number of bytes received, 
number of packets received, average end-to-end delay and the time at which first packet is being received for DSR, AODV and ZRP. Their result showed that ZRP was not up to the task and it performed poorly throughout all the simulation sequences. Their work did not include DYMO protocol and used fixed mobility speed of 1-8 mps and pause time but they used different network sizes with different nodes.

G. Sharma, et.al [2], analyzed the performance of AODV, DSR and DYMO under the effect of two shadowing model, as Constant and Lognormal. Used well known network simulator Qualnet 5.2, They took 75 nodes with different maximum speed and analyzed various performance parameter such as throughput, number of bytes received, average end-to-end delay. They observed that for constant model AODV outperformed the other two protocols. DSR showed the worst performance. But for the log-normal, DSR showed better performance than AODV and DYMO.

P. K. Maurya, et.al [13] compared ZRP, AODV, DYMO and DSR using Qualnet 5.2. They analyzed the throughput, average jitter, average end-to-end and packet delivery ratio in two different phases. First phase was used to analyze pause times and in second phase they varied the maximum speed of nodes in the scenarios. ZRP had lower throughput, lower PDR than AODV, DSR and DYMO and made itself out of the race. In the second phase AODV gave better performance than DYMO and ZRP but lower than DSR. Overall, AODV performed better under different network conditions.

Subramanya, et.al [14] compared proactive (OLSR), reactive (AODV, DSR, LAR) and hybrid (ZRP) routing protocols for stationary and mobile nodes by varying the node density $(25,50,75,100,150,200$ and 250) using Qualnet 5.0.2 network simulator. They considered AODV, DSR, LAR, and OLSR, ZRP routing protocols for analysis and varied nodes numbers along with mobility speed. They took parameters such as throughput, average jitter, average end-to-end delay and packet delivery ratio for the analysis.

\section{OVERVIEW OF ROUTING PROTOCOLS} 3.1 Dynamic Source routing protocol (DSR)

The DSR protocol [7,2, and 10] is a reactive routing protocol, but the concept used is explicit source routing. In this routing protocol data packet which are sent contains the complete, sequenced array of all the nodes via which each data packet has to go through to reach the destination [2]. DSR allows the network to be completely self-organizing and self-configuring, without the need for any pre-existing network infrastructure. DSR is composed of two mechanisms of Route Discovery and Route Maintenance [7]. When a mobile node has a packet to send to some destination, it first checks its route cache to decide whether it already has a route to the destination. If it has an unexpired route, it will use this route to send the packet to the destination. On the other hand, if the cache does not have such a route, it initiates route discovery by broadcasting a route request packet. Each node receiving the route request packet searches throughout its route cache for a route to the intended destination. If no route is found in the cache, it adds its own address to the route record of the packet and then forwards the packet to its neighbors. This request propagates through the network until either the destination or an intermediate node with a route to destination is reached. Whenever route request reaches either to the destination itself or to an intermediate node which has a route to the destination, a route reply is unicasted back to its originator. Route is maintained by using route error packets and acknowledgment.

\subsection{Optimized Link State Routing (OLSR)}

OLSR $[8,14]$ is a proactive and table driven routing protocol. It uses periodic messages to update the topological information of the network among the nodes. The nodes exchange this information to establish a route to the destination node in the network. The advantage of this scheme is that routes are immediately available at each node to the destination node [14].It reduces the possible overhead in the network protocol and are used to multipoint relays (MPR). Reducing the time interval for the control messages transmission brings more reactivity to the topological changes $[8,14]$.OLSR protocols is suitable for large and dense area of networks. OLSR uses two kinds of the control messages namely hello and topology control. Hello messages are used for finding the information about the link status and the host's neighbours [14].

\subsection{Zone Routing Protocol (ZRP)}

The Zone Routing Protocol (ZRP) [9, 10, 13, and 15] combines both reactive routing and pro-active routing protocols into a hybrid routing protocol [15]. ZRP is proposed to reduce the control overhead of proactive routing protocols and decrease the latency caused by routing discover in reactive routing protocols [10]. ZRP defines a zone around each node consisting of its k-neighborhood (e. g.k=3). In ZRP, the distance and a node, all nodes within hop distance from node belongs to the routing zone of node [9]. ZRP is formed by two sub-protocols, a proactive routing protocol: Intra-zone Routing Protocol (IARP) [9, 13] is used inside routing zones and a reactive routing protocol: Inter-zone Routing Protocol (IERP) $[9,13]$ is used between routing zones, respectively. The Inter-zone Routing Protocol (IERP) [9, 13] is used to communicate between nodes of different routing zones. It is a reactive routing protocol and the route discovery process is only initiated when needed or on demand. This makes route finding slower, but the delay can be minimized by use of the Bordercast Resolution Protocol (BRP).

\section{SIMULATION SETUP}

Simulations is carried out on QualNet version 6.1[12]. In this paper we have evaluated the performance variation of MANET Routing Protocols DSR,OLSR and ZRP by changing the pause time of nodes and packet transmission rate with which it can move in the network, over an area of $750 \times 750 \mathbf{m}^{2}$.Among various nodes application of Constant Bit Rate is applied. All the nodes in the depicted scenario are given a mobility using the protocol of Random waypoint mobility model. Simulation parameters are shown in table.1 and simulation results are shown in figures from 1 to 8 . With 
the help of simulation results we have analyzed Average Jitter, Packet delivery ratio, throughput, and End-to-End delay for the given protocol. These parameters are defined below:

\subsection{Packet delivery ratio}

Packet delivery ratio is the ratio of total packets sent by the source node to the successfully received packets by the destination node.

\subsection{Throughput}

It is defined as the information in bits which is received successfully by the destination in an average time. Its unit is bit per seconds.

\subsection{Average End-to-End delay}

It is the time elapsed when a packet is sent from the source node and is successfully received by the destination node. It includes delays as delay for route discovery, propagation time, data transfer time, and intermediate queuing delays.

\subsection{Average Jitter}

Jitter is the time variation between subsequent packet arrivals; it is caused by network congestion, timing drift, or route changes. For an efficient protocol, it must be as low as possible.

Table.1 Simulation Parameters

\begin{tabular}{|l|l|}
\hline \multicolumn{1}{|c|}{ Parameter } & \multicolumn{1}{c|}{ Value } \\
\hline Simulation time & 120 seconds \\
\hline Channel frequency & $2.4 \mathrm{GHz}$ \\
\hline MAC protocol & 802.11 \\
\hline Physical layer Radio-type & $802.11 \mathrm{~b}$ \\
\hline Packet size & 512 bytes \\
\hline Transport layer protocol & UDP \\
\hline Traffic source & CBR \\
\hline Routing Protocols & DSR,OLSR,ZRP \\
\hline Pathloss Model & Two Ray \\
\hline No. of nodes & 60 \\
\hline Shadowing Model & Constant \\
\hline Maximum Speed(mps) & 10 \\
\hline Minimum Speed & $0 \mathrm{mps}$ \\
\hline Pause Time(sec) & $(10,20.25,30)$ and 30 \\
\hline $\begin{array}{l}\text { Rate of transmission of } \\
\text { packet }\end{array}$ & $\begin{array}{l}5 \text { and }(2,10,15,20) \text { packet } \\
\text { per second }\end{array}$ \\
\hline
\end{tabular}

\section{RESULTS AND DISCUSSION}

Fig.1 shows that variation of throughput against pause time. It can be observed that DSR throughput is almost same but OLSR has throughput increasing with varying pause time and it is decreased for 10sec pause time and ZRP has lowest throughput but for $25 \mathrm{sec}$ pause time ZRP throughput increases.

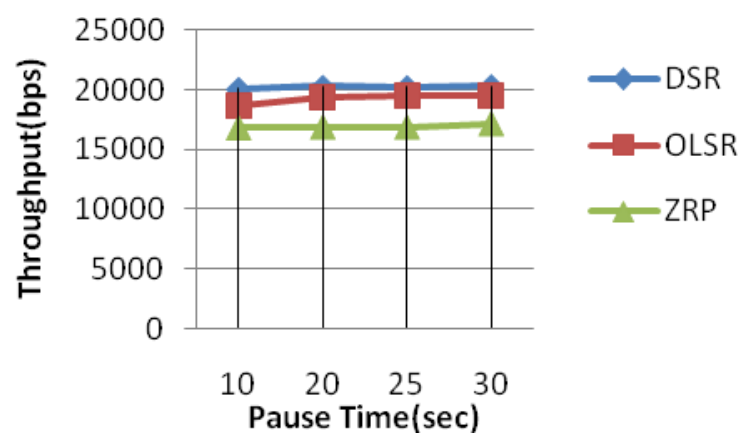

Fig.1 Throughput vs Pause Time

Fig.2 shows the variation of average jitter against pause time. It is observed that OLSR performs well at $10 \mathrm{sec}$ and for 20, 25 and $30 \mathrm{sec}$ pause time it decreases. ZRP jitter variation is constant but ZRP performs worst than DSR .DSR average jitter is almost of constant value but for 30 sec pause time it decreases.

Fig.3, shows the variation of average end to end delay against pause time of nodes and it is observed that for ZRP the average end-to-end delay is constant, but OLSR shows worst performance at 20 and $30 \mathrm{sec}$ pause time.DSR shows decrease in end-to-end delay with varying pause time

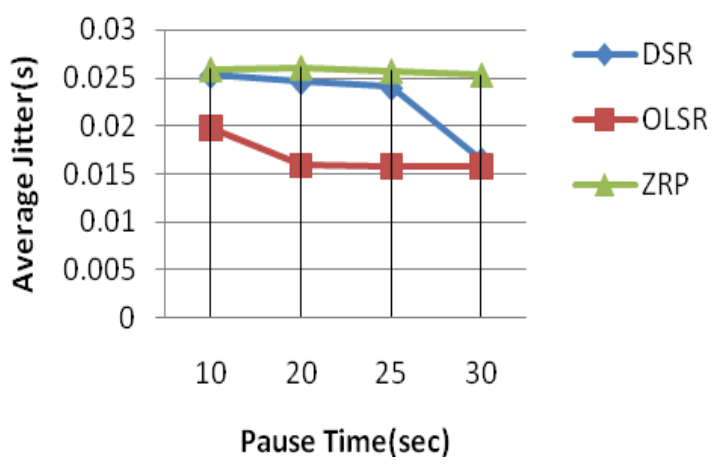

Fig.2 Average Jitter vs Pause Time

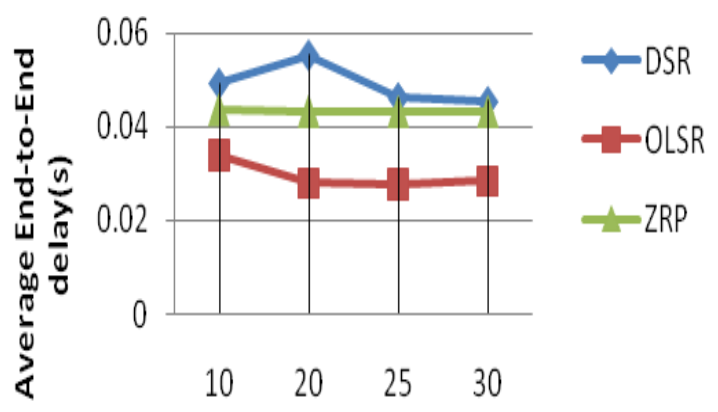

Pause time(sec)

Fig.3 Average end-to-end delay vs. Pause Time 


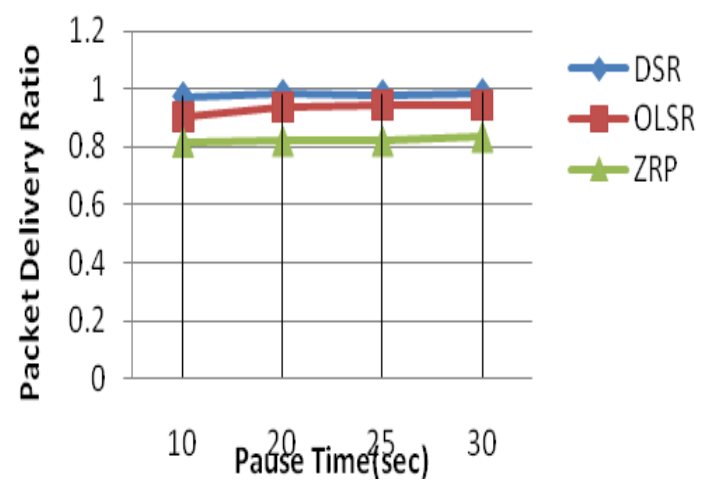

Fig.4 Packet delivery Ratio vs Pause Time

Fig. 4, shows the variation of pause time against packet delivery ratio. It is observed that DSR packet delivery ratio is of almost constant value but ZRP shows the lowest packet delivery ratio performance.OLSR shows increase in Packet delivery ratio with increase in pause time.

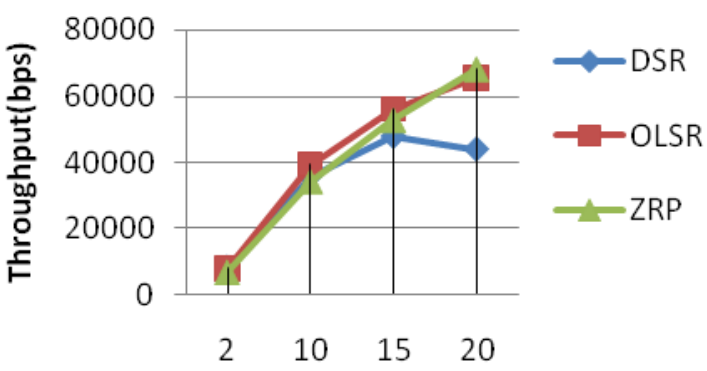

Packet Transmission Rate(Packet/sec)

.Fig.5 Throughput vs Packet Transmission Rate

Fig.5 shows that variation of throughput against packet transmission rate, it can be observed that DSR and OLSR throughput increases when packet transmission rate increases .OLSR shows better throughput than ZRP.ZRP has lowest throughput and it is decreasing when the packet transmission rate increase for 20 packet per second.

Fig.6 shows the variation of average jitter against packet transmission rate. It is observed that DSR performed worst and jitter was increasing when there was increase in the packet transmission rate. OLSR has lesser average jitter than ZRP and DSR but OLSR jitter increased for 20 packet transmission rate.ZRP showed increase in the average jitter other than ZRP average jitter increased when there is increase in the packet transmission rate.

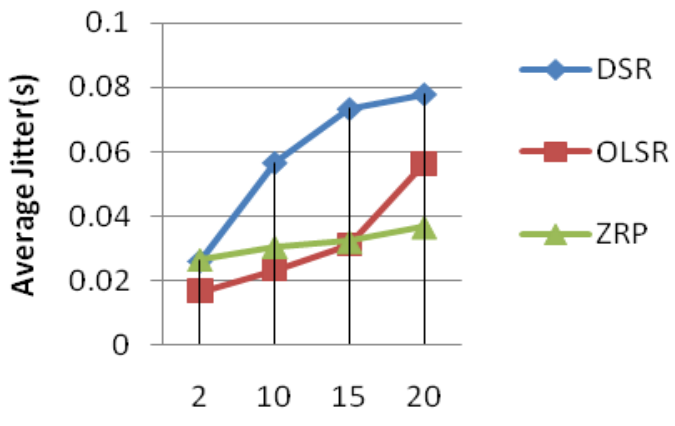

Packet Transmission Rate(Packet/sec)

\section{Fig.6 Average Jitter vs Packet Transmission Rate}

Fig.7, shows the variation of average end to end delay against packet transmission rate of nodes and it is observed that OLSR and ZRP has same value of average end-to-end delay and it increases with packet transmission rate, but DSR shows worst performance when packet transmission rate increases.

Fig. 8 shows the variation of packet delivery ratio against packet transmission rate. It can be observed that DSR packet delivery ratio decreases when there is increase in the packet transmission rate but DSR shows worst packet delivery ratio performance for 20 per second. OLSR packet delivery ratio is better than ZRP and it decreases when there is increase in the packet transmission rate and ZRP has lowest packet delivery ratio and it shows almost constant value of packet delivery ratio and ZRP shows better packet delivery ratio than DSR at 15 and 20 packet transmission rate.

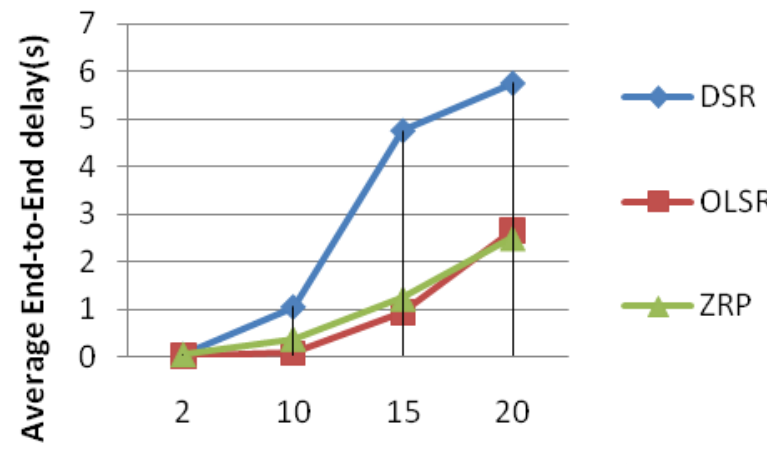

Packet Transmission Rate(Packet/sec)

Fig.7 Average end-to-end delay vs Packet Transmission

Rate 


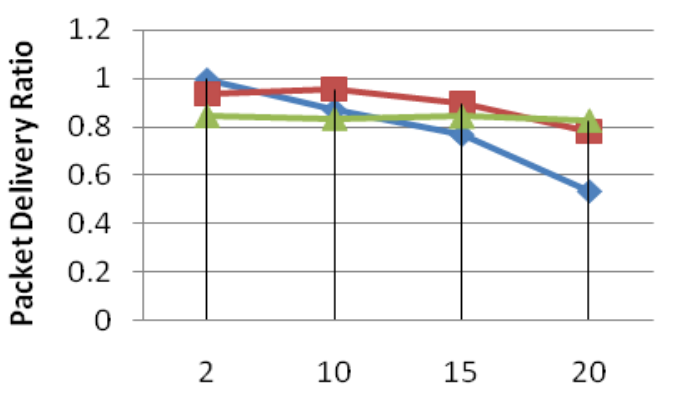

Packet Transmission Rate(Packet/sec)

Fig.8 Packet delivery ratio vs Packet Transmission Rate

\section{CONCLUSIONS}

In this paper, performance comparison of DSR, OLSR and ZRP routing protocol for mobile ad-hoc networks with variable pause time and packet transmission rate is presented. We measure End to End delay (s), Average Jitter (s), Throughput and Packet delivery ratio as performance metrics. Our simulation results show that DSR performs well for throughput and packet delivery ratio for pause time compared to OLSR and ZRP. ZRP performs worst in throughput and packet delivery ratio with varying pause time. DSR shows increase in throughput for all values and decrease in packet delivery ratio for all value with varying packet transmission rates.DSR performs worst for average end to end delay in all network conditions. Among all protocols,DSR for packet transmission rate shows worst performance when packet rate is increased. OLSR throughput and packet delivery ratio is better than ZRP with varying pause time and ZRP shows lowest throughput and packet delivery ratio with varying pause time. OLSR shows best performance for average jitter and average end-to-end delay with the varying pause time and packet transmission rate. ZRP shows better throughput and packet delivery ratio than DSR with varying packet transmission rate.OLSR for packet transmission rate is better than ZRP and DSR.

\section{REFERENCES}

[1] V. Sahu, G. Sharma, and R. Paulus, "Study of Performance Variation of AODV Routing Protocol for Various Parameter Patterns using Qualnet Simulator", volume-54, no. 6, pp. 7-11, September, 2012, IJCA (0975-8887).

[2] Gaurav Sharma ,V. Sahu,P.K. Maurya, R.Paulus ," Analyzing the Effect of Constant and Lognormal Shadowing Model on Ad-hoc Routing Protocols" Volume 66- No.8,IJCA,March,pp-16-19,Mar 2013.

[3] P.K. Maurya, Gaurav Sharma ,V. Sahu, R. Paulus," Performance Measurement and Analysis of AODV and DYMO Routing Protocols for MANET under Free Space and Two Ray Propagation Models" Volume 65No.8,IJCA,March,pp-8-11,Mar 2013.

[4] C.E. Perkins and E.M. Royer, "Ad-hoc On-Demand Distance Vector Routing”, Proceeding of 2nd IEEE Workshop, Mobile Computing System Applications, pp:90-100, 1999.
[5] Perkins, E. Belding-Royer, and S. Das, "Ad hoc OnDemand Distance Vector (AODV) Routing, "draft-ietfmanet-aodv-13.txt, Feb. 2003.

[6] I.Chakeres, C.Perkins, "Dynamic MANET On-Demand (DYMO) Routing," IETF Internet-Draft, draft-ietfmanet-dymo-23, pp. 20-25, October.2012.

[7] J.Broch, D. Jhonson, and D.Maltz, "The dynamic source routing protocol for mobile adhoc networks for IPv4" IETF RFC 4728, Feb 2007.

[8] P.Jaquet,P. Muhlethaler and Aqayyum. "Optimized Link State Routing Protocol”, IETF Draft, draft-ietf-manetolsr-06.txt.

[9] Z J. Haas, M. R. Pearlman, and P. Samar, "The Zone Routing Protocol (ZRP) for Adhoc Networks", draftietf-manet-zone-zrp-04.txt.July 2002.

[10] S. R. Raju and J.Mungara"Performance Evaluation of ZRP over AODV and DSR in Mobile Adhoc Networks Using Qualnet'ISSN 1450-216X Vol.45 No.4 (2010), pp.651-667, European Journal of Scientific Research, 2010.

[11] Mario Gerla, Xiaoyan Hong, Guangyu Pei," Fisheye State Routing Protocol (FSR)", IETF, Draft, draft-ietfmanet-fsr-03.txt, 2002.

[12] The QualNet simulator, www.scalable-networks.com.

[13] Prashant kumar Maurya, Rajeev Paulus,A.K. Jaiswal ,Mahendra Srivastava , "Performance Analysis of ZRP over AODV, DSR and DYMO for MANET under Various Network Conditions using QualNet Simulator" IJCA,March.2013.

[14] Subramanya Bhat.M, Shwetha.D and Devaraju.J.T"A Performance Study of Proactive, Reactive and Hybrid Routing Protocols using Qualnet Simulator "Volume 28-No.5, IJCA. Aug. 2011.

[15] K.Suresh and K.Jogendra "Comparative Performance Study of Zone Routing Protocol over AODV and DSR Routing Protocols on Constant Bit Rate (CBR)" Volume 45- No.4, May 2012, IJCA (0975 - 8887).

[16] Aditi Agrawal, Rajeev Paulus ,A.K Jaiswal, Alok Singh,'Performance Comparison of Routing Protocols Based on Different Models in Mobile Adhoc Network", International Journal of Scientific and Research Publications, Volume 2, Issue 7, ISSN 22503153, July 2012.

[17],R.Paulus,Priyanka.D.Kumar,Prinu.c.Philip,Anil Kumar,"Performance Measurement and Analysis of AODV and DYMO Routing Protocols for MANET under Free Space and Two Ray Propagation Models", Volume 65- No.8,IJCA,March,pp-8-11,Mar 2013. 